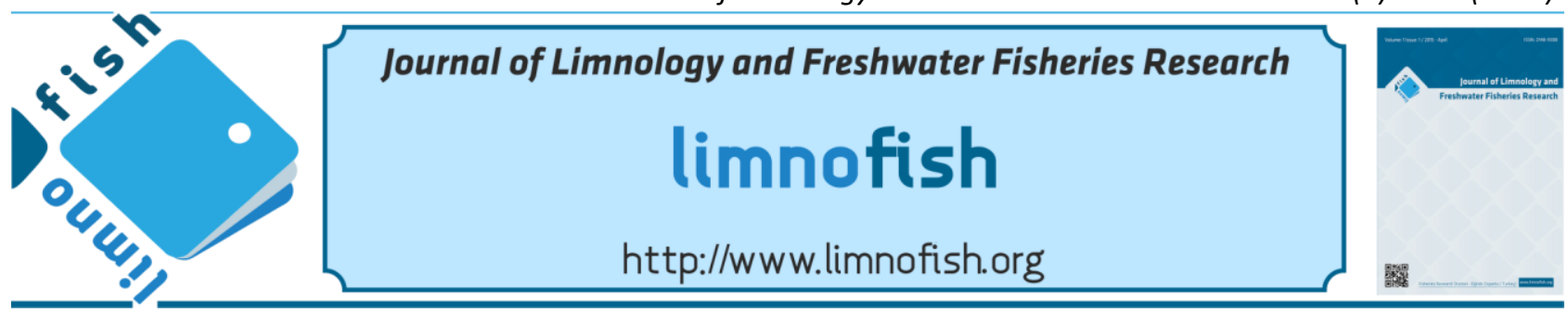

\title{
Ontogenetic Diet Shift of Invasive Gibel Carp (Carassius gibelio, Bloch 1782) in Karamenderes River (Turkey)
}

\author{
Nurbanu PARTAL ${ }^{* *}$ (D), Sükran YALÇIN ÖZDILLEK ${ }^{2}$ (D) \\ ${ }^{1}$ Çanakkale Onsekiz Mart University, Natural and Applied Sciences, 17100 Çanakkale-Turkey \\ ${ }^{2}$ Çanakkale Onsekiz Mart University, Faculty of Arts and Science, Department of Biology, 17100 Çanakkale-Turkey
}

\section{A B STRACT}

The ontogenetic diet shift of invasive Carassius gibelio (Bloch, 1782) was investigated in Karamenderes River, Turkey. The fieldwork was performed during summer 2012, autumn 2012 and spring 2013. The fishes were caught by electrofishing and using gill nets. Nine fork length groups were used in order to assess the ontogenetic diet shift. The gut contents were assessed by the index of relative importance that was calculated from the frequency of occurrence, numerical abundance, and volumetric analyses. The most abundant length groups of $C$. gibelio were $18-20 \mathrm{~cm}, 6-8 \mathrm{~cm}$, and $27-29 \mathrm{~cm}$ length groups during summer 2012, autumn 2012 and spring 2013, respectively. The feeding intensity was the lowest in the length groups of $15-17 \mathrm{~cm}$ during summer 2012, in 3-5 cm length group in autumn 2012 and in $24-26 \mathrm{~cm}$ length group in spring. Seasonal variations were observed in the ontogenetic diet shift of $C$. gibelio. Large specimens consumed more animal materials during summer and more algae in autumn. There was not any significant niche overlap recorded between small and large specimens except summer. Any niche overlap between small and large specimens might be advantageous for the establishment success of invasive Gibel carp in Karamenderes River.

Keywords: Gut contents, feeding intensity, diet overlap, IRI

\section{ARTICLE INFO}

\section{RESEARCH ARTICLE}

Received : 20.09.2018

Revised : : 10.12.2018

Accepted : :12.12.2018

Published : 25.04.2019

DOI:10.17216/LimnoFish.461758

\section{* CORRESPONDING AUTHOR}

nurbanupartal@gmail.com

Phone : +905383433477

Karamenderes Çayı’nda (Türkiye) İstilacı Gümüşi Havuz Balığının (Carassius gibelio, Bloch 1782) Beslenmesindeki Ontogenetik Değişim

Öz: Bu çalışmada Karamenderes Çayı'nda bulunan istilacı Carassius gibelio (Bloch, 1782) türünün beslenmesindeki ontogenetik değişimin belirlenmesi amaçlanmıştır. Arazi çalışmaları Yaz 2012, Sonbahar 2012 ve İlkbahar 2013 mevsimlerinde gerçekleştirilmiştir. Balıkların yakalanmasında elektroşoker ile çeşitli ağlar kullanılmıştır. Beslenmedeki ontogenetik değişimi belirlemek için, balıklar çatal boylarına göre dokuz gruba ayrılmıştır. Balıkların sindirim kanalı içerikleri besin bulunma sıklığı, sayısal bolluk ve hacimsel oran kullanılarak nisbi önem indeksi ile hesaplanmıştır. C. gibelio bireylerinin mevsimlere göre bol olan boy grupları sırasıyla Yaz $2012(18-20 \mathrm{~cm})$, Sonbahar $2012(6-8 \mathrm{~cm})$ ve İlkbahar $2013(27-29 \mathrm{~cm})$ şeklindedir. Beslenme şiddeti Yaz 2012'de 15-17 cm, Sonbahar 2012'de 3-5 cm ve İlkbahar 2013'de 24-26 cm boy gruplarında en az olduğu belirlenmiştir. $C$. gibelio bireylerinin ontogenetik beslenme alışkanlığında zamansal ve mekânsal olarak farklılıklar gözlenmiştir. Büyük bireyler yaz mevsiminde daha çok hayvansal besin ve sonbahar mevsiminde alglerle beslendiği ve küçük bireylerle büyük bireylerin besinleri arasında yaz mevsimi haricinde önemli bir çakışma olmadığı belirlendi. Küçük ve büyük bireylerin besinleri arasında herhangi bir çakışmanın olmaması, gümüşi havuz balığının Karamenderes'de yerleşme başarısı için bir avantajı olabilir.

Anahtar kelimeler: Sindirim kanalı içeriği, beslenme şiddeti, diyet çakışması, IRI

How to Cite

Partal N, Yalçın Özdilek Ş, 2019. Ontogenetic Diet Shift of Invasive Gibel Carp (Carassius gibelio, Bloch 1782) in Karamenderes River (Turkey). LimnoFish. 5(1): 6-16. doi: 10.17216/LimnoFish.461758

\section{Introduction}

Carassius gibelio (Bloch, 1782) is one of the major invasive species which was introduced to Trace region first in the 1980s (Özulug et al. 2004; Ilhan et al. 2005) and spreaded over many freshwater systems rapidly throughout Turkey (Aydin et al.
2011; Ekmekçi et al. 2013). In general, this species is known as a generalist, it has opportunistic omnivorous feeding strategy and feeds on different foods in different environments (Sakai et al. 2001; Gaygusuz et al. 2006; Ekmekçi et al. 2013). It is obvious that high variety in food resources of this 
invasive species will affect many other indigenous species living in the same habitat (Goodell et al. 2000) and make it more advantageous among competitors. In addition to having advantages of this species in the interspecific relationship, the high variety of food resources may change during ontogeny and the intraspecific resource partitioning may another advantage of this species in the introduced ecosystems. There are many studies about ecological traits (Lockwood et al. 2013; Ekmekçi et al. 2013; Tarkan 2013), gut contents (Specziár et al. 1997; Rybczyk 2006; Y1lmaz et al. 2008; Rogozin et al. 2011; Partal 2014; Partal and Yalçın Özdilek 2017) and feeding characteristics (Specziár et al. 1997; Rybczyk 2006; Yılmaz et al. 2008; Rogozin et al. 2011; Partal 2014; Yalçın Özdilek and Jones 2014; Partal and Yalçın Özdilek 2017) of this species. C. gibelio has been first recorded in Karamenderes river which is on the Northwestern part of Turkey in 2007 (Yalçın Özdilek 2008). There are some records on the feeding habits of $C$. gibelio from Karamenderes river (Yalçın Özdilek and Jones 2014; Partal and Yalçın Özdilek 2017), however, there is a gap in the knowledge about ontogenetic diet shift of this species. There is a limited study on the ontogenetic diet shift of $C$. gibelio and the data on this subject with the ontogenetic niche overlap and trophic position will serve to understand the establishment success of this species.

The morphological, physiological and behavioral changes during the developmental stage may result in ontogenetic diet shift (Wilbur 1980; Miller and Rudolf 2011; De Roos and Persson 2013; Nakazawa 2015). In addition, changes in foraging ability depending on the growth may cause the ontogenetic diet shift in fish (Bergman and Greenberg 1994; Jeppesen et al. 2003; Alcaraz and García-Berthou 2007; Nakazawa 2015). Shifting in feeding pattern is common with a function of age and length in many animal species (Wilbur 1980; Miller and Rudolf 2011; De Roos and Persson 2013; Nakazawa 2015). Data on the ontogenetic diet shift is very important for evaluating the ecological role of a species (Werner and Gilliam 1984; Post 2003). Intraspecific competition reduces the population growth particularly in the limited resource condition (Bolnick et al. 2011). The data on the ontogenetic diet shift of invasive $C$. gibelio may serve to take some measures related to mitigating the adverse effects of this invasive species. We aimed to reveal the food diversity in different length groups and the ontogenetic diet shift of C. gibelio quantitatively in this study. This study will serve to understand if this species has such advantage make it successive in establish and spread.

\section{Materials and Methods \\ Study area and sampling}

Karamenderes River, about $109 \mathrm{~km}$ in length, originates from the Kaz and A $\breve{g} 1$ Mountains and directs to West and North and flows into Çanakkale strait after watering Kumkale plate in Biga Peninsula, Çanakkale. There are two reservoirs along the river. One is in the Bayramiç province, which is about 86.5 cubic hectometer water capacity, and the other is in Pınarbaşı village, which is smaller than the other. These reservoirs are used for irrigation purposes (Figure 1).

C. gibelio is first recorded in Karamenderes at the lower part of the Pınarbaşı village after fish stocking studies on Bayramiç Dams by the activities of Directorate of National Water Affairs (Yalçın Özdilek 2008). The samplings were conducted in three seasons, during summer 2012 (July-August 2012), autumn 2012 (October-November 2012), and spring 2013 (May 2013). Sampling could not be performed in winter because of inconvenient weather conditions for sampling. The fish sampling has performed at 14 stations along the Karamenderes River from the upper parts of the dams to the river mouth. The names of the stations from up to down are Karaköy 1, Karaköy 2, Evciler, Evciler trout farm, Çırpılar, Mollahasanlar, Bayramiç-Çan road, Ahmetçeli, Sarmısaklı, Pınarbaşı, Kalafat, Kumkale bridge (3), Kumkale closed end (2), Kumkale open end (1) (Figure 1).

Different sampling device was used for fishing according to habitat characteristics. Fish were collected by scanning about $20 \mathrm{~m}$ lengths of the river during 20 minutes in every station by electrofishing (SAMUS) on the upper sites of Karamenderes. Gill nets in different mesh size $(18 \mathrm{~mm}, 22 \mathrm{~mm}, 25 \mathrm{~mm}$, and $32 \mathrm{~mm}$ knot to knot) and different lengths (160 m-2.5 m, $100 \mathrm{~m}-2 \mathrm{~m}, 15 \mathrm{~m}-2 \mathrm{~m}$, and $30 \mathrm{~m}-1 \mathrm{~m}$ ) were used for fishing (average of 24 hours) in the river mouth stations. In addition, fyke net composed of 837 sets each has $12 \mathrm{~m}$ long and a cast net, which has $10 \mathrm{~mm}$ mesh size and its radius $140 \mathrm{~cm}$ were used for fishing in some stations. All the fish samples were transferred to the laboratory in an icebox and after identification, they preserved in a deep freeze with labeled.

\section{Laboratory procedures}

The gut contents of 215 specimens of totally 251 specimens could be examined. Before dissection, the fork length and weight were measured by the standard ruler $( \pm 0.01 \mathrm{~mm})$ and a balance $( \pm 0.1 \mathrm{~g})$. After dissection, the total gut length from the 
esophagus to the anus was measured using the same ruler. The sex of the specimens was determined under a stereomicroscope. The gut contents were evacuated in a graduated cylinder, which contains $70 \%$ ethanol. The total gut volumes were measured by the replacement of ethanol level.

Diluted gut contents in a Sedgewick- Rafter lam were examined under a stereomicroscope x10 magnitude. The number and sizes (Sun and Liu 2003) of each food category were recorded after the description of the taxon at the possible level.

The percentage of empty guts, vacuity index (VI $\%$ ), were used to assess the feeding intensity (Hureau 1966). The feeding intensity was assessed taking into consideration the length groups, sex, season and stations. Vacuity Index (VI) was used for calculating the feeding intensity by using

$V I=$ empty gut number $\mathrm{x} 100 /$ total gut number equation (Hureau 1966; Costa and Cabral 1999).

The percentage of the relative index (IRI \%) was evaluated to assess gut contents data using the frequency of occurrence $(F \%)$, numerical $(N \%)$ and volumetric $(V \%)$ methods (Pinkas et al. 1971; Prince 1975; Hyslop 1980).
$I R I=(N \%+V \%) \times F \%$

$F_{i} \%=i$ prey items frequency of occurrence in the gut $\mathrm{x} 100 /$ total number of full guts

$N_{i} \%=i$ prey items total number $\mathrm{x} 100 /$ prey items total number

$V_{i} \%=i$ prey items total volume $\mathrm{x} 100 /$ prey items total volume

$$
\begin{gathered}
H=-\sum p_{i} \ln p_{i} \\
p_{i}=N_{i} / N
\end{gathered}
$$

equation. The trophic level was calculated as

$$
T L_{k}=1+\left(\sum_{j=1}^{11} P_{j} \times T L_{j}\right)
$$

in the equation (Cortés 1999). The IRI \% values of each food category were used to calculate the diversity and trophic position.

Fish were grouped into nine-length class categories and the differences in IRI \% value of each food category in each length group were tested by nonparametric Kruskal Wallis test.

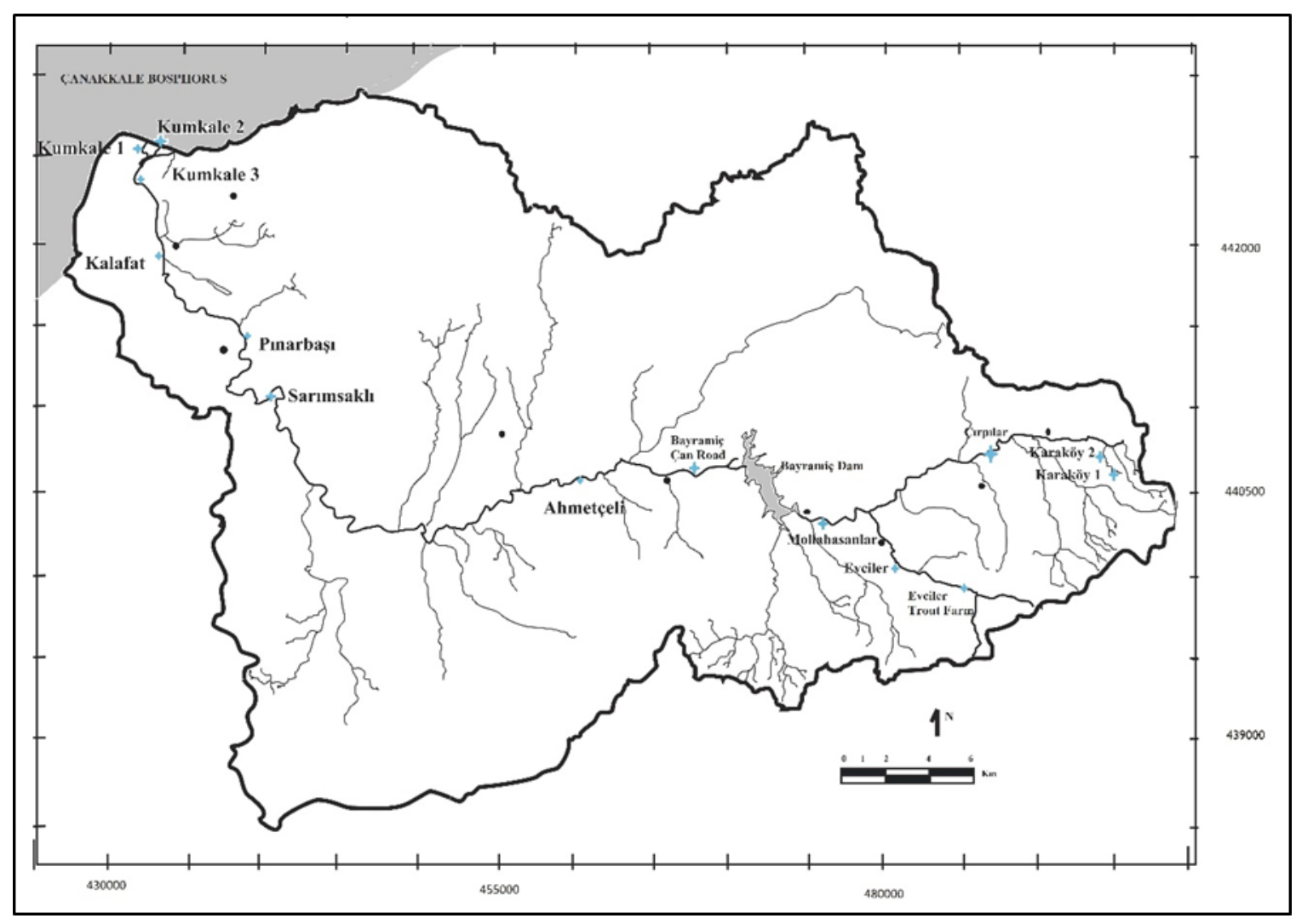

Figure 1. Sampling area (Partal and Yalçın Özdilek 2017 (adapted)).

\section{Results}

A total of 215 specimens were all caught at the lower stations of Bayramiç Reservoir. There were no specimens encountered at the upper stations of this dam. $62 \%, 27 \%$ and $12 \%$ of the specimens were collected during summer 2012, autumn 2012 and spring 2013, respectively. The spatial and seasonal relative abundance of specimens indicates that 
the most abundant specimens were recorded at the Kumkale River mouth station in summer, Ahmetçeli station in autumn and Kumkale bridge (3) station in Spring with the percentages of 60.9, 14.9 and 16.1, respectively (Figure 2). The most abundant length group was $6-8 \mathrm{~cm} F L$ with the percentage of 16.7 in total. The $18-20 \mathrm{~cm}, 6-8 \mathrm{~cm}$, and $27-29 \mathrm{~cm} \mathrm{FL}$ groups were the most abundant length groups in summer, autumn and spring seasons, respectively (Figure 3).

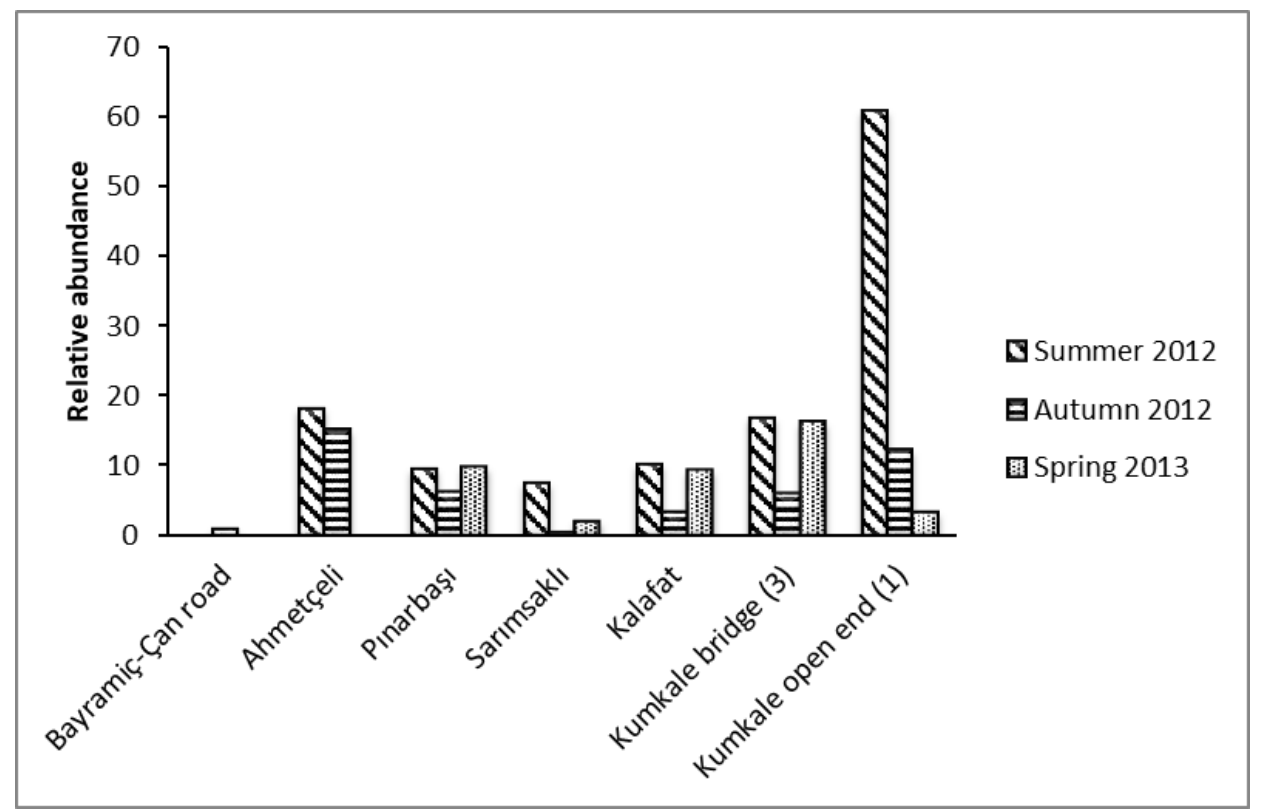

Figure 2. The distribution and relative abundance of $\mathrm{C}$. gibelio specimens according to seasons and the station.

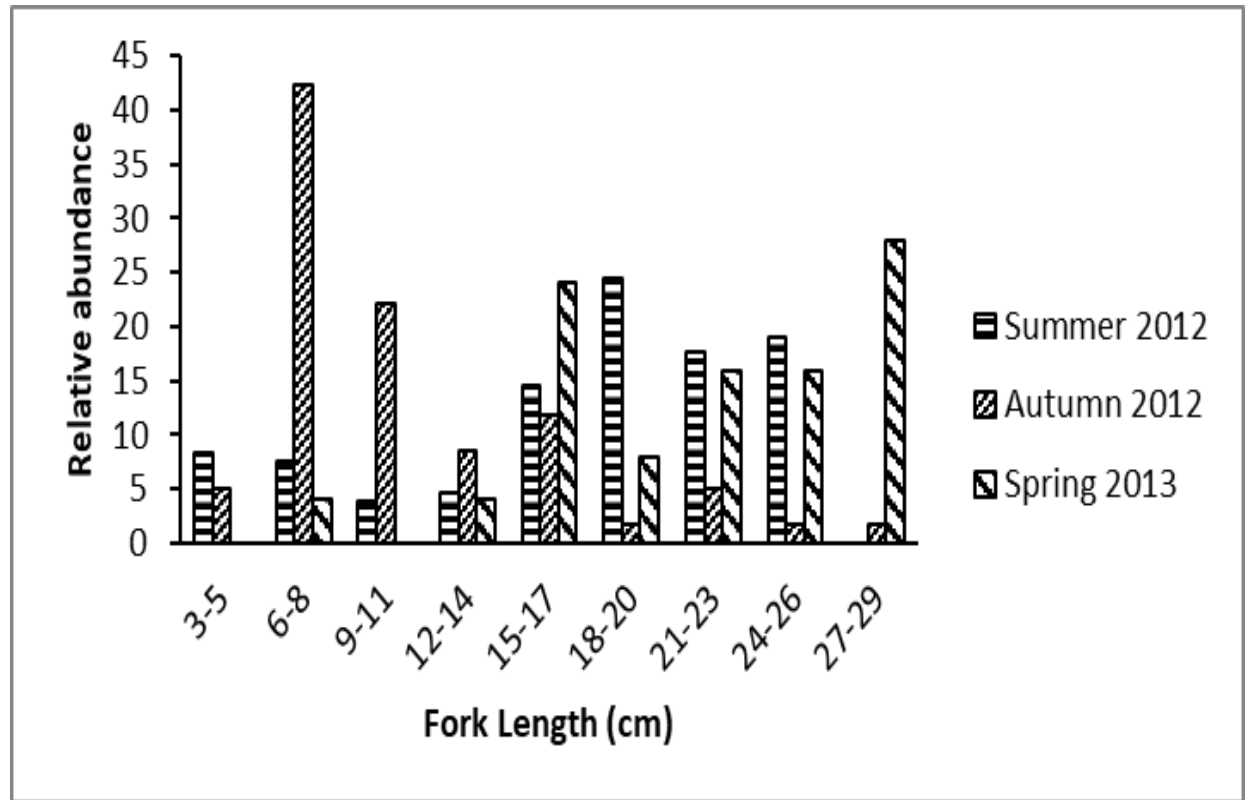

Figure 3. The distribution and relative abundance of the length groups according to the seasons.

Table 1. Shannon-Wiener Diversity Index (SWI) and Vacuity Index (VI) values according to the length groups.

\begin{tabular}{|c|c|c|c|c|c|c|c|c|c|c|c|c|c|c|c|c|c|c|}
\hline \multirow{3}{*}{$\begin{array}{c}\text { Sampling } \\
\text { Season }\end{array}$} & \multicolumn{18}{|c|}{ Length Groups $(\mathrm{cm})$} \\
\hline & \multicolumn{2}{|c|}{$3-5$} & \multicolumn{2}{|c|}{$6-8$} & \multicolumn{2}{|c|}{$9-11$} & \multicolumn{2}{|c|}{$12-14$} & \multicolumn{2}{|c|}{$15-17$} & \multicolumn{2}{|c|}{$18-20$} & \multicolumn{2}{|c|}{$21-23$} & \multicolumn{2}{|c|}{$24-26$} & \multicolumn{2}{|c|}{$27-29$} \\
\hline & SWI & VI & SWI & VI & SWI & VI & SWI & VI & SWI & VI & SWI & VI & SWI & VI & SWI & VI & SWI & VI \\
\hline Summer 2012 & 2.23 & 9.1 & 2.51 & 0 & 2.34 & 20 & 2.46 & 33.3 & 1.76 & 55.6 & 2.26 & 37.5 & 1.86 & 34.8 & 1.39 & 44 & - & - \\
\hline Autumn 2012 & 1.69 & 33.3 & 2.05 & 0 & 2.23 & 23.1 & 1.78 & 20 & 1.56 & 14.3 & 0.89 & 0 & 1.7 & - & 1.47 & 0 & 1.57 & 0 \\
\hline Spring 2013 & - & - & 1.39 & 0 & - & - & 1.41 & 0 & 2.19 & 16.7 & 1.64 & 0 & 2.04 & 0 & 1.99 & 25 & 1.63 & 0 \\
\hline
\end{tabular}




\section{Gut Contents}

The gut contents of $C$. gibelio consisted of siliceous algae, green algae, vascular plants, pine pollen, amphipods and chironomids (Table 2). Algae took an important part in the gut contents by frequency and abundance. As members of Bacillariophyceae, Navicula sp., Fragilaria sp. and partly Cocconeis sp. taxa were dominant organisms in nearly all gut contents. Some animal groups such as Oligochaeta members could not include in the gut content analysis due to rapid digestion. However, there were encountered
Oligochaeta setae in nearly every size group in every season.

When the gut contents were grouped as four-diet categories (detritus, periphyton, macrophyte, and macroinvertebrate) periphyton dominate nearly all length groups except $15-20 \mathrm{~cm}$ length groups in autumn according to N\% values (Figure 4). Particularly, macroinvertebrates were low values both abundance and volume in $>20 \mathrm{~cm}$ length specimens in autumn. The $\mathrm{V} \%$ values of macroinvertebrates were low in $<15 \mathrm{~cm}$ and $>20 \mathrm{~cm}$ specimens in spring and autumn, respectively.

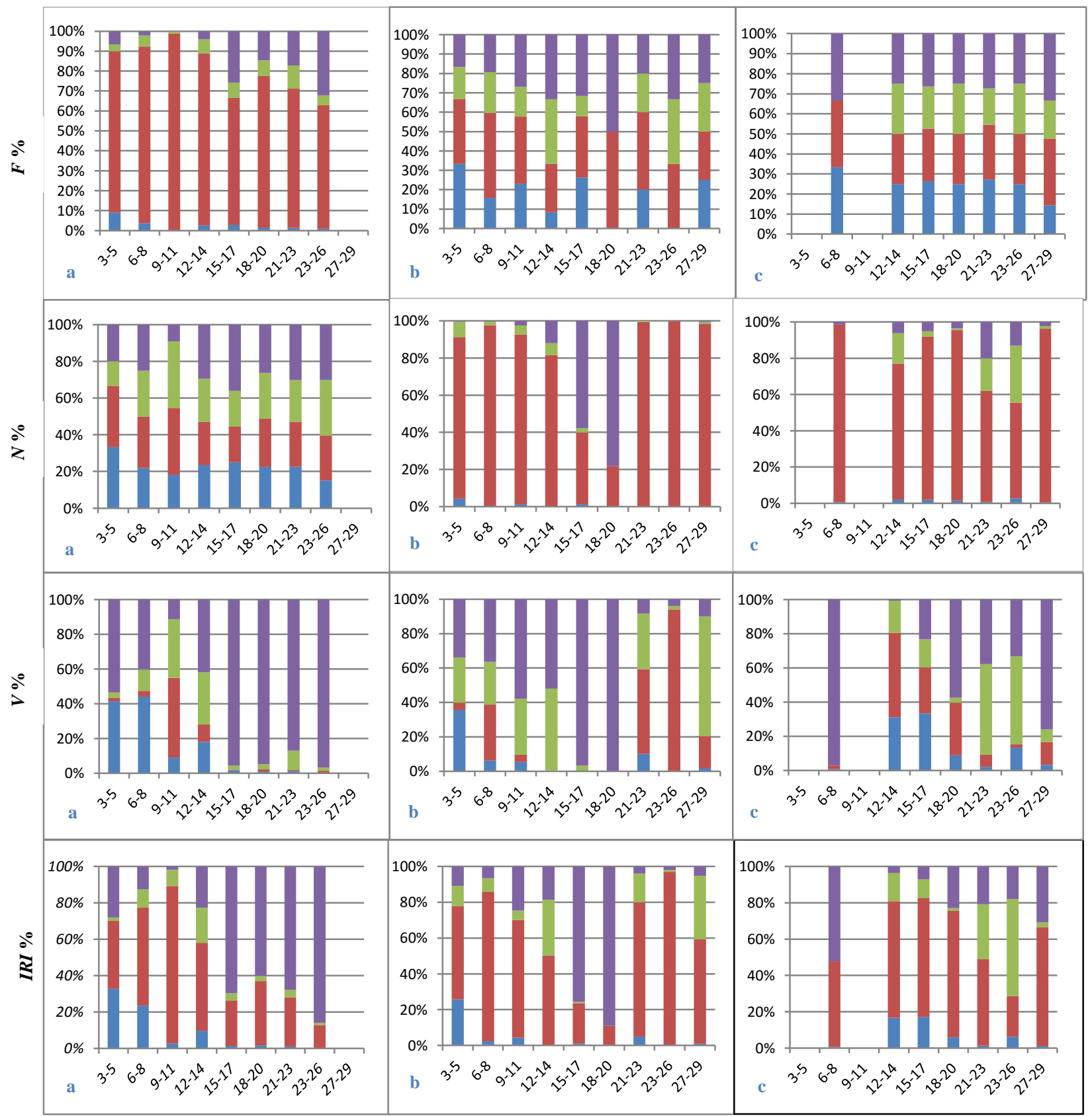

Fifure 4. The occurrence (F \%), number ( $\%$ ), volume (V \%) and Index of Relative Importance (IRI \%) of prey items in the gut contents, in terms of the length groups and seasons (a: Summer 2012; b: Autumn 2012; c: Spring 2013. Blue: detritus; red: periphyton; green: macrophyte; purple: macroinvertebrate) 
Table 2. Seasonal (SUM: Summer 2012; A: Autumn 2012; SP: Spring 2013) IRI \% values of length groups.

\begin{tabular}{|c|c|c|c|c|c|c|c|c|c|c|c|}
\hline & \multirow[b]{3}{*}{ Prey Items } & \multicolumn{10}{|c|}{ Seasonal IRI \% Values of Length Groups } \\
\hline & & \multicolumn{2}{|c|}{ 3-5 } & \multicolumn{3}{|c|}{$6-8$} & \multicolumn{2}{|c|}{$9-11$} & \multicolumn{3}{|c|}{$12-14$} \\
\hline & & SUM & $\mathbf{A}$ & SUM & $\mathbf{A}$ & SP & SUM & $\mathbf{A}$ & SUM & $\mathbf{A}$ & SP \\
\hline Cyanobacteria & Oscillatoria sp. & & 1.82 & & 14.14 & & & 2.33 & & & \\
\hline \multirow{20}{*}{ 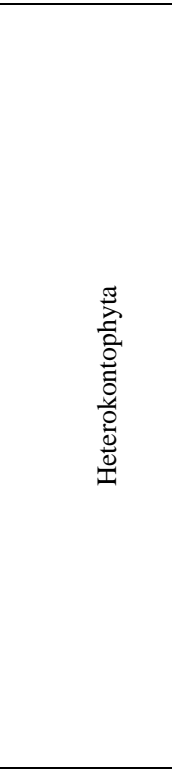 } & Amphora sp. & 1.19 & 0.33 & 1.29 & 0.23 & & 2.34 & 2.70 & 0.40 & 0.80 & \\
\hline & Cocconeis sp. & 0.34 & & 3.90 & 1.34 & 2.0 & 7.30 & 10.25 & 2.50 & 10.45 & 2.8 \\
\hline & Cyclotella sp. & & & & & & & & 0.19 & & \\
\hline & Cymatopleura sp. & 0.17 & & 0.74 & 0.08 & & 0.10 & & 0.45 & & \\
\hline & Cymbella sp. & 0.22 & & 5.26 & 0.19 & 0.7 & 15.65 & 1.17 & 5.54 & 3.52 & \\
\hline & Diatoma sp. & 0.04 & & 0.09 & 0.06 & & 0.01 & & & & \\
\hline & Fragilaria sp. & 0.01 & 0.91 & 0.07 & 8.64 & & 0.08 & & 0.07 & 0.16 & \\
\hline & Fragilaria sp. (chain) & 13.48 & 0.54 & 17.49 & 1.26 & 16.8 & 2.37 & 15.04 & 18.58 & 0.24 & 54.8 \\
\hline & Gomphonema sp. & 0.35 & 1.95 & 0.12 & 3.96 & & & 2.18 & 0.13 & & \\
\hline & Gyrosigma sp. & 0.34 & 0.40 & 0.25 & 0.07 & 0.7 & 12.07 & 1.20 & 4.18 & 0.66 & \\
\hline & Licmophora sp. & & 1.44 & & 6.70 & & & 1.10 & & & \\
\hline & Melosira sp. & 5.82 & & 8.79 & 0.30 & & 12.49 & 0.93 & 5.95 & 2.44 & \\
\hline & Navicula sp. & 8.98 & 41.69 & 11.54 & 43.19 & 15.4 & 16.94 & 28.45 & 6.71 & 31.54 & 6.1 \\
\hline & Neidium sp. & & & & & & & & 0.06 & & \\
\hline & Nitzschia sp. & 2.88 & & 1.73 & & & 16.55 & & 0.15 & & \\
\hline & Pinnularia sp. & & 2.34 & 0.004 & 0.76 & & & 0.05 & 0.20 & 0.16 & \\
\hline & Rhoicosphaenia sp. & 2.28 & 0.33 & 0.94 & 0.40 & & 0.07 & 0.05 & 0.11 & & \\
\hline & Stephanodiscus sp. & 0.03 & 0.35 & 0.41 & 0.003 & & & & 1.23 & 0.16 & \\
\hline & Ulnaria sp. & 0.95 & & 1.10 & 0.07 & 10.7 & 0.02 & 0.26 & 0.66 & & \\
\hline & Vaucheria sp. & & & & 0.31 & & & & & & \\
\hline \multirow{16}{*}{ 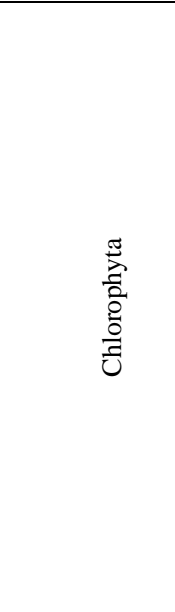 } & Ankistrodesmus sp. & & & & & & & & & & 0.6 \\
\hline & Chlorella sp. & & & & 1.53 & & & & & & \\
\hline & Chlorophyta & & & 0.03 & & & & & & & \\
\hline & Cladophora sp. & 1.10 & & 2.06 & 0.56 & & 2.70 & 0.46 & 6.80 & & \\
\hline & Closterium sp. & 0.19 & & 0.06 & 0.15 & 0.8 & 0.70 & & & & \\
\hline & Conjugatophyceae & & & & & & & & & & \\
\hline & Cosmarium sp. & 0.02 & & 0.02 & & & & & 0.02 & & \\
\hline & Microspora sp. & & & & & & & & & & \\
\hline & Oedogonium sp. & 0.01 & & 0.19 & 0.05 & & 3.51 & 0.03 & 0.16 & 0.25 & \\
\hline & Pandorina sp. & & & & & & & & & & \\
\hline & Pediastrum sp. & & & 0.10 & 0.04 & & & & 1.08 & & \\
\hline & Scenedesmus sp. & 0.01 & & & 0.02 & & & 0.06 & & & \\
\hline & Spirogyra sp. & & & & 5.78 & & & 1.002 & & & \\
\hline & Stigeoclonium sp. & 0.39 & 0.73 & 0.06 & 0.99 & & 0.33 & 0.61 & 0.38 & & 0.7 \\
\hline & Ulothrix sp. & & & & 0.01 & & & & & & \\
\hline & Zygnema sp. & & & & & & & 0.14 & & & \\
\hline \multirow{18}{*}{ 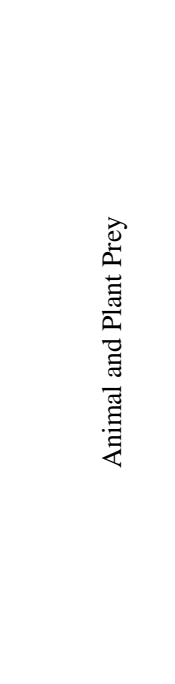 } & Amphipoda & 23.94 & & 7.99 & 5.79 & & 1.49 & 18.23 & 19.50 & 9.73 & \\
\hline & Chironomidae & 0.11 & & & & 52.3 & & 0.25 & & & \\
\hline & Copepoda & & 11.04 & & & & & & & 0.56 & \\
\hline & Crustacea & & & & 0.29 & & & & & & \\
\hline & Insecta & 3.91 & & 2.46 & & & & & & & \\
\hline & Keratella sp. & & & & & & & & 0.46 & & \\
\hline & Nematoda & & & & & & & & & & \\
\hline & Ostracoda & & & 1.94 & 0.53 & & & 6.21 & 2.72 & 8.31 & \\
\hline & Plant (seed) & & & & 0.01 & & & & & & \\
\hline & Pollen & 0.20 & 0.27 & 0.04 & 0.004 & & 0.02 & & & & \\
\hline & Plant & 0.23 & 10.15 & 7.59 & 0.13 & & 2.12 & 0.13 & 11.98 & & 14.8 \\
\hline & Fish egg & 0.04 & & 0.10 & 0.04 & & & & 0.03 & & 3.6 \\
\hline & Animal Detritus & 15.78 & & 19.07 & & & & & 0.80 & & 15.5 \\
\hline & Digested Detritus & 0.41 & & 0.02 & & & 0.32 & & & & \\
\hline & Other organisms & 16.58 & 25.71 & 4.41 & 2.39 & 0.7 & 2.44 & 4.37 & 8.97 & 0.13 & 1.2 \\
\hline & Cystic material & & & & 0.001 & & & & 0.02 & & \\
\hline & Bryozoa & & & 0.03 & & & 0.38 & 2.82 & & 30.87 & \\
\hline & Cladocera & & & 0.10 & & & & & & & \\
\hline
\end{tabular}


Table 2. (Continous)

\begin{tabular}{|c|c|c|c|c|c|c|c|c|c|c|c|c|c|c|c|}
\hline & \multirow[b]{3}{*}{ Prey Items } & \multicolumn{14}{|c|}{ Seasonal IRI \% Values of Length Groups } \\
\hline & & \multicolumn{3}{|c|}{ 15-17 } & \multicolumn{3}{|c|}{ 18-20 } & \multicolumn{3}{|c|}{ 21-23 } & \multicolumn{3}{|c|}{ 24-26 } & \multicolumn{2}{|c|}{ 27-29 } \\
\hline & & SUM & $\mathbf{A}$ & SP & SUM & $\mathbf{A}$ & SP & SUM & $\mathbf{A}$ & SP & SUM & $\mathbf{A}$ & SP & $\mathbf{A}$ & SP \\
\hline \multirow{3}{*}{$\begin{array}{l}\text { Cyano- } \\
\text { bacteria }\end{array}$} & Anabaena sp. & & & & 0.003 & & & & & & & & & & \\
\hline & Merismopedia $\mathrm{sp}$. & & & & 0.02 & & & & & & & & & & \\
\hline & Oscillatoria sp. & 0.001 & & 1.28 & 0.002 & & & & & & & & & & \\
\hline \multirow{21}{*}{ 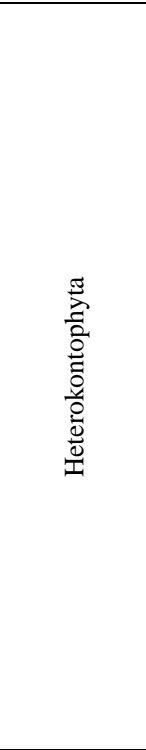 } & Amphora sp. & 3.94 & 3.65 & 0.02 & 3.29 & 1.82 & & 2.35 & & 0.07 & 0.01 & & & 0.17 & 0.1 \\
\hline & Cocconeis sp. & 1.49 & 10.53 & 0.29 & 7.03 & 3.64 & 3.1 & 6.96 & 5.18 & 2.72 & 2.66 & 5.96 & 0.5 & & 0.5 \\
\hline & Cyclotella sp. & 0.01 & & & & & & & & & & & & & \\
\hline & Cymatopleura sp. & 0.09 & 0.03 & 0.32 & 0.03 & & 0.2 & 0.15 & & 0.42 & 0.01 & & & 0.23 & \\
\hline & Cymbella sp. & 0.34 & 1.22 & 2.02 & 6.38 & & & 3.09 & 0.27 & 0.37 & 0.23 & 12.8 & & 1.48 & 0.3 \\
\hline & Diatoma sp. & 0.01 & & 0.36 & 0.003 & & & 0.01 & & & 0.001 & & & & \\
\hline & Epithemia sp. & & & & 0.07 & & & & & & & & & & \\
\hline & Fragilaria sp. & & 0.09 & 0.33 & 0.14 & & & 0.001 & & & & & & & \\
\hline & Fragilaria sp. (chain) & 0.04 & 1.51 & 28 & 3.11 & 1.84 & 45.5 & 0.76 & 6.38 & 37.5 & 5.02 & 4.32 & 6.4 & 1.22 & 21.7 \\
\hline & Gomphonema sp. & 0.01 & 0.21 & & 0.08 & & & 0.04 & 0.11 & & 0.02 & & & & \\
\hline & Gyrosigma sp. & 2.15 & 0.28 & 1.65 & 2.56 & & & 2.28 & 1.04 & & 0.06 & 0.79 & & 1.25 & \\
\hline & Melosira sp. & 0.49 & 0.84 & 8.73 & 2.85 & & & 1.63 & 16.39 & 5.36 & 0.93 & 34.9 & 4.9 & 27.8 & 37.3 \\
\hline & Meridion sp. & & & & 0.004 & & & & & & 0.003 & & & & \\
\hline & Navicula sp. & 8.7 & 3.34 & 16.6 & 5.32 & 1.82 & 15.3 & 6.34 & 44.46 & 0.86 & 1.12 & 38.2 & 6.4 & 26.1 & 3.2 \\
\hline & Nitzschia sp. & 1.32 & & 0.03 & 0.76 & & & 1.31 & & & 0.29 & & & & 0.02 \\
\hline & Pinnularia sp. & 0.01 & 0.11 & & 0.02 & & & 0.003 & & & 0.002 & & & & 0.03 \\
\hline & Rhizosolenia sp. & & & & 0.0001 & & & & & & & & & & \\
\hline & Rhoicosphaenia sp. & 0.72 & 0.72 & & 0.10 & 1.82 & 2.03 & 0.39 & 0.87 & & 0.06 & & & & \\
\hline & Stephanodiscus sp. & 0.13 & & 0.01 & 0.60 & & & 0.03 & & & 0.01 & & 1.6 & & 0.2 \\
\hline & Surirella sp. & & & & 0.001 & & & & & & & & & & \\
\hline & Ulnaria sp. & 5.46 & & 5.90 & 2.80 & & 3.5 & 1.66 & 0.16 & 0.38 & 1.95 & & 2.5 & & 2.3 \\
\hline \multirow{17}{*}{ 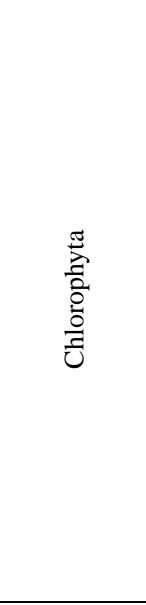 } & Ankistrodesmus sp. & 0.003 & & & 0.001 & & & & & & & & & & \\
\hline & Chaetomorpha sp. & & & & 0.02 & & & & & & & & & & \\
\hline & Chlorophyta & & & & & & & 0.03 & & & 0.01 & & & & \\
\hline & Cladophora sp. & 0.01 & & & 1.04 & & & 2.22 & & 3.35 & 0.54 & & & & \\
\hline & Closterium sp. & 0.003 & & 0.03 & 0.01 & & & 0.02 & & & & & & & \\
\hline & Conjugatophyceae & & & & 0.10 & & & 0.26 & & 9.26 & 0.01 & & 7.3 & & 0.2 \\
\hline & Cosmarium sp. & 0.01 & & & 0.09 & & & 0.003 & & & 0.01 & & & & \\
\hline & Microspora sp. & 0.13 & & & 0.13 & & & 0.08 & & & 0.07 & & & & \\
\hline & Oedogonium sp. & 0.01 & & & 0.51 & & & 0.05 & 15.81 & 0.66 & 0.01 & & 3.4 & 1.65 & \\
\hline & Pandorina sp. & & & & 0.002 & & & & & & & & & & \\
\hline & Pediastrum sp. & 0.02 & & & 0.005 & & & & 0.15 & & & & & & \\
\hline & Scenedesmus sp. & & 0.03 & & 0.001 & & & 0.001 & & & 0.0004 & & & & \\
\hline & Spirogyra sp. & & & 0.09 & & & & 0.01 & & 3.29 & & & & & 1.4 \\
\hline & Stigeoclonium sp. & 0.02 & 0.08 & & 0.24 & & & 0.07 & 0.18 & & 0.03 & & & 0.21 & \\
\hline & Ulothrix sp. & & & & 0.35 & & & 0.003 & & & & & & & \\
\hline & Ulvales & & & & & & & 0.05 & & & & & & & \\
\hline & Zygnema sp. & & & & 0.01 & & & 0.15 & & & & & & & \\
\hline Charophyta & Mougetia sp. & & & & 0.01 & & & & & & & & & & \\
\hline & Amphipoda & 51.62 & 38.70 & 0.38 & 21.45 & 76.1 & 0.9 & 52.42 & 4.04 & 0.46 & 59.97 & 2.003 & 13.9 & 0.22 & 27.5 \\
\hline & Chironomidae & 0.91 & 0.0003 & 2.26 & 1.34 & & 21.3 & 0.25 & & & & & & 4.87 & 1.8 \\
\hline & Copepoda & 0.52 & 0.0003 & 0.27 & 2.02 & 0.02 & & 0.27 & & & 0.10 & & & & \\
\hline & Crustacea & 0.12 & 0.11 & & 0.18 & & & 0.80 & & & 3.83 & & & & \\
\hline & Gastropoda & 0.001 & & & 0.01 & & & & & & 0.08 & & & & \\
\hline ১্ర & Insecta & 0.03 & 0.26 & 0.36 & 0.38 & & & 0.20 & & 9.35 & 0.55 & & & & 0.5 \\
\hline$\stackrel{A}{=}$ & Nematoda & & & & 0.003 & & & 0.0001 & & & & & & & \\
\hline$\frac{\vec{E}}{\bar{A}}$ & Ostracoda & 16 & 36.47 & & 34.48 & 12.9 & & 13.79 & & & 20.89 & & & & 1.03 \\
\hline$\vec{\sigma}$ & Pollen & 0.005 & & 0.01 & 0.11 & & 0.1 & 0.17 & & & 0.25 & 1.12 & & & \\
\hline $\bar{z}$ & Plant & 3.80 & 0.14 & 10.1 & 0.38 & & 1.4 & 0.95 & & 13.7 & 0.39 & & 42.8 & & 0.98 \\
\hline$\stackrel{\mathscr{G}}{\Xi}$ & Fish egg & 0.45 & & 3.65 & 0.28 & & 0.7 & 0.08 & & 10.9 & 0.47 & & 3.96 & 0.11 & 0.04 \\
\hline & Animal Detritus & 0.01 & & 1.67 & 0.002 & & & 0.001 & & & & & 3.7 & & \\
\hline & Digested Detritus & 0.77 & & & 0.07 & & 1.97 & 0.23 & & 1.19 & 0.14 & & 1.6 & & \\
\hline & Other organisms & 0.59 & 1.09 & 15.5 & 1.58 & & 3.9 & 0.86 & 4.95 & 0.15 & 0.20 & & 0.9 & 1.02 & 1.1 \\
\hline & Plant (circle shaped) & 0.06 & & & 0.03 & & & 0.001 & & & 0.06 & & & & \\
\hline & Cystic material & & & 0.23 & 0.01 & & & 0.02 & & & & & & 0.12 & \\
\hline & Bryozoa & & 0.56 & & & & & & & & & & & 33.5 & \\
\hline
\end{tabular}




\section{Ontogenetic diet shifts}

The ontogenetic diet shift indicated seasonal variation taking into consideration IRI \% values. There was a significant difference among $I R I \%$ of various length groups in all three seasons $(X=27.003, \quad P<0.01, \quad d f=7 ; \quad X=20.603, \quad P<0.05$, $d f=8 ; X=14.073, P<0.05, d f=6)$. Heterekontophyta members were the most common food groups in nearly all length groups. While the small specimens $(3-4 \mathrm{~cm})$ feed on mostly Heterokontophyta members, the larger specimens consumed mostly animal foods such as Amphipoda and Ostracoda in all the seasons. Particularly, the IRI \% value of Amphipoda members was more than $50 \%$ in the large specimens in summer. Navicula sp. was the highest IRI \% in the $3-11 \mathrm{~cm} F L$ group in autumn. According to three season data, the critical length group shifting diet from the herbivorous to carnivore dominated feeding strategy is $12 \mathrm{~cm} \mathrm{FL}$. However, the IRI \% of
Amphipoda members were $23.9 \%, 18.2 \%$, and Copepoda members $11.0 \%$ in autumn, Chironomidae members were high $(52.3 \%)$ in spring in smaller than $12 \mathrm{~cm} F L$ group (Table 2).

\section{Relative gut length and feeding strategy}

The mean relative gut length of $C$. gibelio is $3.47 \pm 0.85$ with the range of 1.22-5.66 (in summer) $2.60 \pm 0.73$ with the range of 1.03-3.84 (in autumn) $3.52 \pm 0.56$ with the range of 2.12-4.56 (in spring). $C$. gibelio specimens have omnivore feeding strategy according to their length groups. (Table 3 ). When group's specimens that were smaller than $12.0 \mathrm{~cm}$ length collected in three seasons combined, the mean $R G I$ value of this combined group was $2.51 \pm 0.77$ that means carnivorous dominant omnivorous feeding strategy. The $R G I$ values of $12.1-17.9 \mathrm{~cm}$ and larger than $18.0 \mathrm{~cm}$ length groups combined in three seasons were $3.38 \pm 0.67$ and $3.66 \pm 0.69$ respectively.

Table 1. Taking into consideration $R G I$ types of feeding in different length groups (O: Omnivorous; H: Herbivorous; C: Carnivorous; N: Number of specimens; GL: Gut length; FL: Fork length; SD: Standart Deviation).

\begin{tabular}{|c|c|c|c|c|c|c|c|c|c|}
\hline Length groups $(\mathrm{cm})$ & $\mathrm{N}$ & Summer (GL/FL \pm SD) & & $\mathrm{N}$ & Autumn (GL/FL \pm SD) & & $\mathrm{N}$ & Spring (GL/FL \pm SD) & \\
\hline $3-5$ & 11 & $2.47 \pm 0.97$ & $\mathrm{O}-\mathrm{C}$ & 3 & $1.55 \pm 0.34$ & $\mathrm{C}-\mathrm{O}$ & - & - & - \\
\hline $6-8$ & 10 & $2.69 \pm 0.43$ & $\mathrm{O}$ & 25 & $2.47 \pm 0.81$ & $\mathrm{C}-\mathrm{O}$ & 1 & 3.16 & $\mathrm{O}$ \\
\hline $9-11$ & 5 & $3.15 \pm 0.92$ & $\mathrm{O}$ & 13 & $2.40 \pm 0.56$ & C-O & - & - & - \\
\hline $12-14$ & 6 & $3.65 \pm 0.75$ & $\mathrm{O}$ & 5 & $2.85 \pm 0.36$ & $\mathrm{O}$ & 1 & 3.13 & $\mathrm{O}$ \\
\hline $15-17$ & 20 & $3.52 \pm 0.73$ & $\mathrm{O}$ & 7 & $3.24 \pm 0.26$ & $\mathrm{O}$ & 6 & $3.21 \pm 0.79$ & $\mathrm{O}$ \\
\hline $18-20$ & 23 & $3.69 \pm 0.74$ & $\mathrm{O}-\mathrm{H}$ & 1 & 3.33 & $\mathrm{O}$ & 2 & $3.67 \pm 0.95$ & $\mathrm{H}-\mathrm{O}$ \\
\hline $21-23$ & 22 & $3.70 \pm 0.78$ & $\mathrm{O}-\mathrm{H}$ & 3 & $3.01 \pm 0.38$ & $\mathrm{O}$ & 4 & $3.58 \pm 0.71$ & $\mathrm{O}$ \\
\hline $24-26$ & 25 & $3.73 \pm 0.76$ & $\mathrm{O}-\mathrm{H}$ & 1 & 3.04 & $\mathrm{O}$ & 4 & $3.81 \pm 0.36$ & $\mathrm{H}-\mathrm{O}$ \\
\hline $27-29$ & - & - & - & 1 & 3.62 & $\mathrm{O}$ & 7 & $3.64 \pm 0.19$ & $\mathrm{O}$ \\
\hline
\end{tabular}

\section{Trophic level}

The trophic level of all $C$. gibelio specimens ranged from 2.03 to 3.34 . The trophic level was low and more or less steady state in spring comparing to summer and autumn. The trophic level is increasing at larger than $12 \mathrm{~cm} F L$ specimens both in the spring and summer seasons. The trophic level of larger than $20 \mathrm{~cm} \quad F L$ specimens decreased dramatically in autumn. The minimum and maximum TLs of all groups are 2.06-3.29, 2.03-3.34 and 2.072.79 in summer, autumn, and spring, respectively (Figure 5).

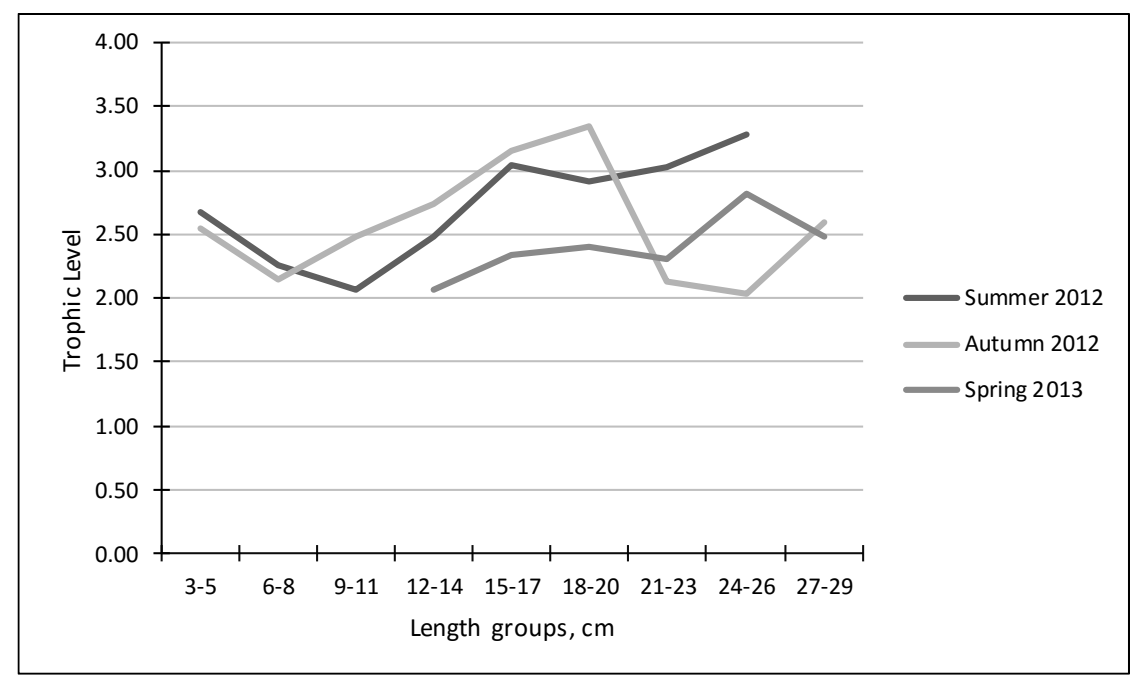

Figure 4. Trophic level of the length groups. 


\section{Discussion}

C. gibelio were observed only the lower part of the Bayramiç Dam along the River Karamenderes. The spatial distribution of $C$. gibelio had seasonal variation along the River Karamenderes. While the specimens larger than $13.6 \mathrm{~cm} F L$ were abundant at the river mouth station, the specimens smaller than $8.8 \mathrm{~cm} F L$ were rich at the upper sites just below the Bayramiç dam in the summer and spring. The smaller specimens might escape from the Bayramiç Dam, which is regularly fished by aquaculture activities and the most available habitat for $C$. gibelio might be at the river mouth conditions.

A previous study based on a gravimetric method indicates that small specimens mostly feed on diatoms and large specimens consume animal materials such as copepods, beetles and chironomids in a Brackish Water Body in Southern Siberia (Rogozin et al. 2011). Another study, which is based on $F \%$ and $N \%$ indicates that small specimens consume zooplanktonic organisms such as copepods and cladocerans in Gelingüllü reservoir, Turkey (Kurankaya 2007). In addition, the phytoplankton was recorded as low frequency comparing to other food organisms in all age groups in Gelingüllü reservoir (Kurankaya 2007). There are no previous study recording gut contents as IRI \% values, however, the results obtained from abundance and frequency in Karamenderes River were apart from the results of the previous records. Periphyton was important diet as frequency and abundance, as well as IRI \%, in almost all length groups.

The amount of animal food might be enough only large specimens who are more capable to collect the animal materials comparing to smaller ones, so the smaller ones have to change their feeding characteristics into algae because of competition. This finding was supported by our high diet diversity in the gut contents of smaller specimens in Karamenderes River. There is no diet diversity in $C$. gibelio feeding patterns in the previous study, and our results indicated a decrease in Shannon diversity in food components of $>12 \mathrm{~cm} \mathrm{C}$. gibelio. This indicated that gibel carp has a wide plasticity in every length group and this wide range on the capability of resource use give them a high advantage for surviving in even very limited resource conditions. In the other views, the food selection of specimens might be related to the abundance of resource users. While the abundance of large Gibel carp specimens were abundant in summer, the small ones were abundant in autumn and spring. In addition, the high amount of large specimens might exploit all favorable animal materials in summer.

In Karamenderes River, feeding intensity of $C$. gibelio had a seasonal variation with high feeding intensity reported by Kirankaya (2007) and Bobori et al. (2012). In general, the feeding intensity estimated based on a number of empty gut indicates seasonal variation, with low in hot summer season because of increasing enzyme activities and digestion metabolism. The seasonal and size depend variation in the feeding intensity might be related with the diet types. For example, in summer the specimens smaller than $8 \mathrm{~cm} T L$ feed mostly on plant materials and likely in autumn the large specimens consumed plant material. It is important that the digestion of plant material is hard when comparing to animal material and the retention duration of plant materials is longer than that of animal materials in the gut (Nikolsky 1978). In addition, the smaller specimens might feed on relatively small food and the retention duration of small animal materials as a diet would be relatively shorter time (Labropoulou et al. 1997) in gut content comparing to large animal materials which are presumably consumed by larger specimens. In other words, the feeding intensity might be explained by fish abundance. In small and large specimens was low abundance in summer and autumn, respectively (Figure 3). This indicates that when the population of C. gibelio low density, they prefer the most available and abundant plant materials as food. The small specimens mostly feed on siliceous algae and probably feed on the mats of periphyton, which are more abundant in the shallow, pooled, high vegetated stony and macrophytes dominant microhabitats along the river. This kind of habitat might be more suitable for small specimens serve them both food and shelter for escaping their predators. Yalçın Özdilek and Jones (2014) stated that the filamentous algae were important food components for about middle size $(13.5-21.1 \mathrm{~cm}$ in FL) $C$. gibelio living in Karamenderes. However, IRI \% results indicate that $12-21 \mathrm{~cm}$ length group members feed on animal prey items in overall stations in Karamenderes river in this study. This study supports that $C$. gibelio is an opportunistic feeder and the plasticity in its feeding strategy might be seasonal and ontogenetic.

C. gibelio has an omnivorous feeding strategy as indicated many studies (Specziár et al. 1997; Balık et al. 2003; Rybczyk 2006; Kirankaya 2007; Y1lmaz et al. 2008; Yalçın Özdilek and Jones 2014; Partal and Yalçın Özdilek 2017). The trophic level based on IRI $\%$ and $R G I$ data support this finding. The ontogenetic diet patterns taking into consideration, there was a conflict between IRI \% and RGI results. Taking into consideration $R G I$ carnivorous dominant feeding strategy was observed in smaller than $12 \mathrm{~cm} F L$ specimens' particularly in summer and autumn and herbivore dominant omnivore strategy was observed in larger than $18 \mathrm{~cm} F L$ specimens particularly in summer and spring. The IRI \% values are indicative 
of instant feeding, so animal materials are digested faster, especially in hot summer and autumn seasons (Windell 1978).

The increase in the trophic level with increasing of the fish length was found in many studies as a natural process (Weber and Brown 2013). In this study, the finding that large specimens have high trophic level in spring and summer is an anticipated result. However, a decrease in the trophic level of larger than $20 \mathrm{~cm}$ in $F L$ might be explained by food availability. During this season because of competition (Yalçın Özdilek 2017) and limited resources (Akbulut et al. 2009), the large specimens may supply their requirements with foods that have lower energy but that are more abundant in surroundings.

\section{Acknowledgements}

This research was supported by TUBITAK 111 Y280 codded project. In field and laboratory studies we thank Ali Rahmi Firat, Sait Gürsoy, Emine İnci Balkan, Gizem Yilmaz and the other team members of the project. This study was presented in FABA14: Symposium on Fisheries and Aquatic Sciences, 25-27 September 2014, Trabzon - Turkey by oral presentation.

\section{References}

Akbulut M, Çelik EŞ, Odabaşı DA, Kaya H, Selvi K, Arslan N, Odabaşı SS. 2009. Seasonal benthic macroinvertebrate community distribution and composition in Menderes Creek, Canakkale, Turkey. Fresen Environ Bull (FEB). 18(11a):2136-2145.

Alcaraz C, García-Berthou E. 2007. Food of an endangered cyprinodont (Aphanius iberus): ontogenetic diet shift and prey electivity. Environ Biol F1sh. 78(3):193-207.

doi: 10.1007/s10641-006-0018-0

Al-Hussaini AH. 1949. On the functional morphology of the alimentary tract of some fish in relation to differences in their feeding habits: anatomy and histology. Q J Microsc Sc1. 3(10):109-139.

Aydın H, Gaygusuz Ö, Tarkan AS, Top N, Emiroğlu Ö, Gürsoy Gaygusuz Ç. 2011. Invasion of freshwater bodies in the Marmara Region (northwestern Turkey) by nonnative Gibel Carp, Carassius gibelio (Bloch, 1782). Turk J Zool. 35(6):829-836.

doi: 10.3906/zoo-1007-31

Balık İ, Karaşahin B, Özkök R, Çubuk H, Uysal R. 2003. Diet of Silver Crucian Carp Carasssius gibelio in Lake Eğirdir. Turk J Fish Aquat Sc. 3:87-91.

Bergman E, Greenberg LA. 1994. Competition between a planktivore, a benthivore, and a species with ontogenetic diet shifts. Ecology. 75(5):1233-1245. doi: $10.2307 / 1937449$

Bobori DC, Salvarina I, Michaloudi E. 2012. Fish dietary patterns in the eutrophic Lake Volvi (East Mediterranean). J Biol Res-Thessalon. 19:139-149.
Bolnick DI, Amarasekare P, Araújo MS, Bürger R, Levine JM, Novak M, Rudolf VHW, Schreiber SJ, Urban MC, Vasseur DA. 2011. Why intraspecific trait variation matters in community ecology. Trends Ecol Evol. 26(4):183-192.

doi: $10.1016 /$ j.tree.2011.01.009

Cortés E. 1999. Standardized diet compositions and trophic levels of sharks. ICES J Mar Sc1. 56(5):707-717. doi: $10.1006 / j m s c .1999 .0489$

Costa MJ, Cabral HN. 1999. Changes in the Tagus nursery function for commercial fish species: some perspectives for management. Aquat Ecol. 33(3):287-292.

De Roos AM, Persson L. 2013. Population and community ecology of ontogenetic development. Princeton, USA: Princeton University Press 538 p.

Ekmekçi FG, Kırankaya ŞG, Gençoğlu L, Yoğurtçuoğlu B. 2013. Türkiye içsularındaki istilacı balıkların güncel durumu ve istilanın etkilerinin değerlendirilmesi. Istanbul University Journal of Fisheries \& Aquatic Sciences. 28:105-140.

Gaygusuz Ö, Gürsoy Ç, Özuluğ M, Tarkan AS, Acıpınar H, Bilge G, Filiz H. 2006. Conversions of total, fork and standart length measurements based on 42 marine and freshwater fish species (from Turkish waters). Turk J Fish Aquat Sc. 6:79-84.

Goodell K, Parker IM, Gilbert GS. 2000. Biological impacts of species invasions: implications for policymakers. In: National Research Council, ed. Incorporating science, economics, and sociology in developing sanitary and phytosanitary standards in international trade. Washington, DC: National Academy Press. p. 87-117.

Hureau JC. 1966. Biologie comparée de quelques poissons Antartique (Nototheniidae). Bulletin de l'institut Oceano graphique (Monaco). 68:1-244.

Hyslop EJ. 1980. Stomach contents analysis. a review of methods and their application. J. Fish Biol. 17:411-429. doi: 10.1111/j.1095-8649.1980.tb02775.x

Ilhan A, Balık S, Sarı HM, Ustaoğlu MR. 2005. Carassius (Cyprinidae, Pisces) species in inland waters of Western and Middle Anatolia, Southern Marmara, Thrace and Western Black Sea Regions and their distributions. Ege Journal of Fisheries and Aquatic Sciences. 22(3-4):343-346.

Jeppesen E, Jensen JP, Jensen C, Faafeng B, Hessen DO, Søndergaard M, Lauridsen $\mathrm{T}$, Brettum $\mathrm{P}$, Christoffersen K. 2003. The impact of nutrient state and lake depth on top-down control in the pelagic zone of lakes: a study of 466 lakes from the temperate zone to the arctic. Ecosystems. 6:313-325. doi: 10.1007/s10021-002-0145-1

Kirankaya SG. 2007. A comperative study on growth, reproduction and feeding biology of mirror carp, Wild Carp (Cyprinus carpio L., 1758) and Prussian Carp [Carassius gibelio (Bloch, 1782)] in Gelingüllü Dam Lake (Yozgat-Turkey) [PhD Thesis]. Hacettepe University. 199 p.

Labropoulou M, Machias A, Tsimenides N, Eleftheriou A. 
1997. Feeding habits and ontogenetic diet shift of the striped red mullet, Mullus surmuletus Linnaeus, 1758. Fish Res. 31(3):257-267. doi: 10.1016/S0165-7836(97)00017-9

Lockwood JL, Hoopes MF, Marchetti MP. 2013. Invasion ecology. John Wiley \& Sons.

Miller TEX, Rudolf VHW. 2011. Thinking inside the box: community-level consequences of stage structured populations. Trend Ecol Evol. 26:457-466. doi: 10.1016/j.tree.2011.05.005

Nakazawa T. 2015. Ontogenetic niche shifts matter in community ecology: a review and future perspectives. Popul Ecol. 57(2):347-354. doi: 10.1007/s10144-014-0448-z

Nikolsky GV. 1978. The Ecology of Fishes. London, England: T.F.H. Publication Inc. Ltd. 352 p.

Özuluğ M, Meriç N, Freyhof J. 2004. The distribution of Carassius gibelio (Bloch,1782) (Teleostei: Cyprinidae) in Thrace (Turkey). Zool Middle East. 31:63-66. doi: 10.1080/09397140.2004.10638023

Partal N. 2014. Distribution and feeding ecology of invasive Carassius gibelio (Bloch, 1782) in Karamenderes Stream, Turkey [Master's Thesis]. Çanakkale Onsekiz Mart University. 89 p.

Partal N, Yalçın Özdilek Ş. 2017. Feeding ecology of invasive Carassius gibelio (Bloch, 1782) in Karamenderes Stream, Turkey. Ege Journal of Fisheries and Aquatic Sciences. 34(2):157-167. doi: 10.12714/egejfas.2017.34.2.07

Pinkas L, Oliphant MS, Iverson ILK. 1971. Food habits of albacore, blue fin tuna, and bonito in California waters. Fish Bull Calif. 152:1-105.

Post DM. 2003. Individual variation in the timing of ontogenetic niche shifts in largemouth bass. Ecology. 84(5):1298-1310.

doi:10.1890/0012-9658(2003)084[1298:IVITTO]2.0.CO;2

Prince ED. 1975. Pinnixid crabs in the diet of young-ofthe-year Copper Rockfish (Sebastes caurinus). Trans. Am. Fish. Soc. 04:539-540. doi: 10.1577/1548-8659(1975)104<539:PCITDO>2.0.CO;2

Rogozin DY, Pulyayevskaya MV, Zuev IV, Makhutova ON, Degermendzhi AG. 2011. Growth, diet and fatty acid composition of Gibel Carp Carassius gibelio in Lake Shira, a brackish water body in Southern Siberia. J Siberian Federal Univ Biol. 4:86-103.

Rybczyk A. 2006. Selected aspects of biological characteristics of the Prussian Carp (Carassius auratus gibelio Bloch, 1783): food, feeding, and condition. Acta Scientiarum Polonorum. Piscaria. 5(2):69-82.

Sakai AK, Allendorf FW, Holt JS, Lodge DM, Molofsky J, With KA, Baughman S, Cabin RJ, Cohen JE, Ellstrand NC, McCauley DE, O'Neil P, Parker IM, Thompson JN, Weller SG. 2001. The population biology of invasive specie. Annual review of ecology and systematics. 305-332.

doi: 10.1146/annurev.ecolsys.32.081501.114037

Shannon CE, Wiener W. 1949. The mathematical theory of communication. Urbana, USA: University of Illinois Press $125 \mathrm{p}$.

Specziár A, Tölg L, Bíró P. 1997. Feeding strategy and growth of Cyprinids in the littoral zone of Lake Balaton. Journal of Fish Biology. 51(6):1109-1124.

doi: 10.1111/j.1095-8649.1997.tb01130.x

Sun J, Liu D. 2003. Geometric models for calculating cell biovolume and surface area for phytoplankton. J Plankton Res . 25(11):1331-1346. doi: 10.1093/plankt/fbg096

Tarkan AS. 2013. Yabancı tatlısu balıklarının dünyada ve Türkiye'de giriş yolları, etkileri ve bunlardan korunma yöntemleri. Istanbul University Journal of Fisheries \& Aquatic Sciences. 28:63-104.

Weber MJ, Brown ML. 2013. Spatiotemporal variation of juvenile common carp foraging patterns as inferred from stable isotope analysis. Transactions of the American Fisheries Society. 142(5):1179-1191. doi: $10.1080 / 00028487.2013 .802253$

Werner EE, Gilliam JF. 1984. The ontogenetic niche and species interactions in size-structured populations. A Rev Ecol Syst. 15:393-425. doi: 10.1146/annurev.es.15.110184.002141

Wilbur HM. 1980. Complex life cycles. Annu Rev Ecol Syst. 11:67-93.

doi: 10.1146/annurev.es.11.110180.000435

Windell JT. 1978. Estimating food consumption rates of fish populations. In T. Bagenal (Ed.), Method for assessment of fish production in freshwaters.. London, England: Blackwell Sci. p. 227-254

Yalçın Özdilek Ş. 2008. Karamenderes'in doğal ve istilacı balıkları. Paper presented at: Ezine İl Değerleri Sempozyumu; Çanakkale, Türkiye.

Yalçın Özdilek Ş. 2017. Seasonal and ontogenetic diet shift of two sympatric cyprinid fish species from the temperate Karamenderes River, Çanakkale, Turkey. Turk J Zool. 41(1):67-81.

doi: 10.3906/zoo-1603-34

Yalçın Özdilek Ş, Jones RI. 2014. The diet composition and trophic position of introduced Prussian Carp Carassius gibelio (Bloch, 1782) and native fish species in a Turkish River. Turk J Fish Aquat Sc . 14:769-776. doi: 10.4194/1303-2712-v14_3_19

Yılmaz M, Bostancı D, Yılmaz S, Polat N. 2008. İki farklı habitatta [Eğirdir Gölü (Isparta) ve Bafra Balık Gölleri (Samsun)] yaşayan Havuz Balığı (Carassius gibelio Bloch, 1782)'nın beslenme rejimlerinin karşılaştırılması. Jounal of Fisheries Science. 2(3):233-240. doi: 10.3153/jfscom.mug.200707 